Portland State University

PDXScholar

Physics Faculty Publications and Presentations

Physics

6-1-1981

\title{
Brownian Motion in a Flowing Fluid Revisited
}

John D. Ramshaw

Portland State University, jdramshaw@yahoo.com

Follow this and additional works at: https://pdxscholar.library.pdx.edu/phy_fac

Part of the Physics Commons

Let us know how access to this document benefits you.

\section{Citation Details}

J.D. Ramshaw, "Brownian motion in a flowing fluid revisited," Phys. Fluids 24, 1210 (1981)

This Article is brought to you for free and open access. It has been accepted for inclusion in Physics Faculty Publications and Presentations by an authorized administrator of PDXScholar. Please contact us if we can make this document more accessible: pdxscholar@pdx.edu. 


\title{
RESEARCH NOTES
}

Research Notes published in this Section include important research results of a preliminary nature which are of special interest to the physics of fluids and new research contributions modifying results already published in the scientific literature. Research Notes cannot exceed two printed pages in length including space allowed for title, abstract. figures, tables, and references. The abstract should have three printed lines. Authors must shorten galley proofs of Research Notes longer than two printed pages before publication.

\section{Brownian motion in a flowing fluid revisited}

\author{
John D. Ramshaw \\ Theoretical Division, University of California, Los Alamos National Laboratory, Los Alamos, New \\ Mexico 87545 \\ (Received 12 January 1981; accepted 11 March 1981) \\ It is shown how the phenomenon of osmosis may be treated using the phenomenological theory of Brownian \\ motion in a flowing fluid. The theory is also generalized to include viscous stresses in the particle and mixture \\ momentum equations.
}

The purpose of this note is to address two questions that have arisen about the phenomenological theory of Brownian motion in a flowing fluid. ${ }^{1}$ The notation is that of Ref. 1, and Eq. (n) from Ref. 1 will be referred to as Eq. $(I-n)$.

The first question is concerned with how the phenomenon of osmosis may be treated within the framework of the theory. Osmosis occurs when a particle-fluid mixture is partitioned by a semi-permeable membrane which acts as a rigid barrier to the particles but through which the fluid may flow freely. In the simplest case, particles are present on one side of the membrane (side 1) but not on the other (side 2). The phenomenon to be explained is this: if the pressure is initially uniform, fluid spontaneously flows from side 2 to side 1 , until an equilibrium is reached in which the pressure on side 1 $\left(p_{1}\right)$ exceeds that on side $2\left(p_{2}\right)$ by the osmotic pressure $q$. In particular, what is the driving force that makes the fluid flow?

No explicit modifications to the theory are needed in order to represent the membrane, because the effect of the latter may be considered part of the force field $G_{p}$. With this in mind, the equilibrium pressure differential is easily obtained by setting $\mathrm{v}, \nabla T$, and $\mathrm{J}_{\text {p }}$ equal to zero in Eqs. (I-9) and (I-15). This yields

$$
\nabla q=\left(1-\alpha_{p}\right) \nabla p-\rho_{f} \mathbf{G}_{f} .
$$

If the Brownian particles are everywhere dilute, so that $\alpha_{p} \ll 1$, then Eq. (1) assumes the simpler form $\nabla(p-q)$ $=\rho_{f} \mathrm{G}_{f}$. Integrating this equation across the membrane, we obtain simply $p_{2}-q_{2}=p_{1}-q_{1}$. (The term $\rho_{f} \mathbf{G}_{f}$ makes a contribution proportional to the membrane thickness; this contribution has been neglected under the assumption that the membrane is very thin.) But $q_{2}=0$ because there are no particles on side 2 ; therefore, $p_{1}=p_{2}+q_{1}$, as was to be shown.

The above argument yields the correct equilibrium pressure differential, but it does not reveal the driving force that sets the fluid in motion initially. To obtain this information, it is necessary to examine the tran- sient equations, in particular, the fluid momentum equation. This equation is not explicitly given in Ref. 1, but is readily found to be

$$
\rho_{f}\left(\partial \mathbf{u}_{f} / \partial t+\mathbf{u}_{f} \cdot \nabla \mathbf{u}_{f}\right)=-\nabla(p-q)-\rho_{\rho} \mathbf{F}+\rho_{f} \mathbf{G}_{f} .
$$

To obtain Eq. (2), use Eq. (I-29) to convert the left member of Eq. (I-9) into $\partial(\rho \mathrm{v}) / \partial t+\nabla \cdot(\rho \mathrm{vv})$. Now replace the approximate momentum flux $\rho \mathrm{vv}$, which is appropriate only when $u_{p}$ and $u_{f}$ are not very different, by the correct momentum flux $\rho_{p} u_{p} u_{p}+\rho_{f} u_{f} u_{f}$. The result then combines with Eqs. (I-2), (I-3), and (I-29) to yield Eq. (2) above. If $G_{f}=0$ and if the particles and fluid are initially at rest with uniform temperature and pressure, then $F$ vanishes and the initial time derivative of $u_{f}$ becomes simply $\partial \mathrm{u}_{f} / \partial t=\left(1 / \rho_{f}\right) \nabla q$. Thus, we see that the gradient of the osmotic pressure $q$ is a driving force in the fluid momentum equation, and that it tends to make the fluid flow in the direction of increasing particle density. Since $q$ is just the pressure in an ideal gas of Brownian particles, it has the for $m$ of an ideal gas partial pressure [cf. Eq. (I-1)]. What is remarkable is that it also has the significance of a partial pressure, even though the particle-fluid mixture is incompressible and is not at all like an ideal gas. This interpretation follows from the fact that $q$ must be subtracted from the total pressure to obtain the pressure whose gradient appears in the fluid momentum equation (2).

It is now clear that the phenomenon of osmosis is correctly and automatically accounted for by the general equations of the theory. These equations can therefore be used in the analysis of transient osmotic effects. The present point of view may also have some pedagogical value, since it provides an essentially mechanical interpretation of the osmotic pressure and a mechanistic route to van't Hoff's law.

We now turn to the second question to be discussed: how would $J_{\phi}$ be affected by the inclusion of viscous stresses in the particle and mixture momentum equations, Eqs. (I-3) and (I-9)? To pursue this ques- 
tion we simply restore the neglected terms. Since the particle-fluid mixture is incompressible, $\nabla \cdot\left[\alpha_{p} u_{p}+(1\right.$ $\left.\left.-\alpha_{p}\right) u_{f}\right]=0$. We are interested only in the limit of large friction, in which $u_{f}$ and $u_{f}$ become very nearly equal to $\mathrm{v}$; the incompressibility condition then implies that $\nabla \cdot \mathrm{v}$ $=\nabla \cdot u_{p}=0$, so the viscous terms may be simplified to their incompressible forms. With this in mind, it is clear that a term $\nabla \cdot\left(\mu_{\phi} s_{\phi}\right)$ must be added to the right member of Eq. $(I-3)$, where $S_{p}=\left(\nabla u_{p}\right)+\left(\nabla u_{p}\right)^{T}$, and the superscript $T$ denotes the transpose. Here, $\mu_{p}$ is the particle shear viscosity, which according to Ref. 1 must be evaluated as though the particles were an ideal gas. If the particles are assumed to be smooth, rigid, and perfectly elastic, then ${ }^{2} \mu_{p}=\left(5 / 64 R^{2}\right)(m k T / \pi)^{1 / 2}=(5 / 32)$ $\left(\rho_{\triangleright}^{0} k T / 3 R\right)^{1 / 2}$. Similarly, a term $\nabla \cdot(\bar{\mu} \mathrm{S})$ must be added to the right member of Eq. $(I-9)$, where $S=(\nabla v)+(\nabla v)^{r}$, and $\bar{\mu}$ is the shear viscosity of the particle-fluid mixture. If the particles are sufficiently dilute, $\bar{\mu}$ is given by the Einstein formula ${ }^{3} \bar{\mu}=\mu\left(1+\frac{5}{2} \alpha_{p}\right)$, where $\mu$ is the shear viscosity of the pure host fluid.

It is now straightforward to repeat the development of Ref. 1, Sec. III with the viscous terms included. The resulting diffusion flux is given by

$$
\begin{aligned}
\mathrm{J}_{p}= & -D \cdot \nabla_{\rho_{D}}-D_{T} \cdot \nabla \ln T+D_{p} \nabla p \\
& +\left(\rho_{p} \rho_{f} / \rho \beta\right)\left(\mathbf{G}_{p}-G_{f}\right)+D_{\nu} \nabla^{2} \mathbf{v},
\end{aligned}
$$

where $D_{p}$ is given by Eq. $(I-19)$ and

$$
\begin{aligned}
& \mathrm{D}=(k T / m \beta) \mathrm{U}+\left(5 \mu \alpha_{p} / 2 \rho \beta\right) \mathrm{s}, \\
& D_{T}=\left(\frac{\rho_{p}}{m \beta}\right)(k T+\eta) \cup+\frac{1}{\beta}\left[\left(\frac{\rho_{\phi} \bar{\mu}}{\rho}\right)\left(\frac{\partial \ln \mu}{\partial \ln T}\right)-\frac{1}{2} \mu_{p}\right] \mathrm{s}, \\
& D_{v}=\mu_{p} / \beta-\left(\rho_{p} \bar{\mu} / \rho \beta\right) ;
\end{aligned}
$$

here $U$ is the unit dyadic, and the dependence of $\mu$ on $p$ has been neglected. Comparison with Eq. (I-15) et seq. shows that the concentration and thermal diffusion coefficients have now become tensors, and an additional term proportional to $\nabla^{2} v$ has appeared. Depending on the values of the various parameters, these modifica- tions may be quantitatively significant even though $v$ varies negligibly over distances of order $R$. [If the latter condition is violated, Eq. (I-4) is no longer adequate, and more extensive modifications become necessary.] For example, the second term in 0 may be neglected relative to the first when $\left(5 \mu \alpha_{p} \bar{v} / \rho L\right) \ll k T / m$, where $L$ is a characteristic length for appreciable spatial variations in $\mathbf{v}(L \gg R)$, and $\bar{v}$ is the associated variation in $|\mathrm{v}|$. For the typical values $\mu / \rho=0.01 \mathrm{~cm}^{2} / \mathrm{sec}$, $\bar{v}=10 \mathrm{~cm} / \mathrm{sec}, L=1 \mathrm{~cm}, T=300 \mathrm{~K}$, and $\rho_{p}^{0}=1 \mathrm{~g} / \mathrm{cm}^{3}$, this condition reduces to $\alpha_{p} R^{3} \ll 2 \times 10^{-14} \mathrm{~cm}^{3}$. For large colloidal particles with $R=10^{-4} \mathrm{~cm}$, the condition becomes $\alpha_{p} \ll 0.02$, which will be satisfied only if the particles are extremely dilute. But for particles with $R$ $=10^{-5} \mathrm{~cm}$, the condition becomes $\alpha_{p} \ll 20$, which is no restriction at all.

Similar numerical estimates may be made for the other new terms in Eqs. (3)-(6), and they further reinforce the conclusion that the a priori neglect of the new terms is hazardous and cannot be recommended as a general procedure. Some or all of these terms will indeed be negligible in many situations, but this must be separately determined in each particular case.

I am grateful to R. S. Hotchkiss and R. T. Foister for their interest and for the stimulating questions which led to this work.

This work was performed under the auspices of the United States Department of Energy.

${ }^{1}$ J. D. Ramshaw, Phys. Fluids 22, 1595 (1979).

${ }^{2} \mathrm{~J}$. O. Hirschfelder, C. F. Curtiss, and R. B. Bird, Molecular Theory of Gases and Liquids (Wiley, New York, 1954), p. 527.

${ }^{3} \mathrm{~J}$. Happel and H. Brenner, Low Reynolds Number Hydrodynamics (Prentice-Hall, Englewood Cliffs, N.J., 1965), p. 441 . 